\title{
Preliminary model comparison for Dynamic Thermal Rating estimation
}

\author{
Emanuele Ogliari, Alfredo Nespoli, Roberto Faranda \\ Department of Energy \\ Politecnico di Milano \\ Milan, Italy \\ \{emanuelegiovanni.ogliari;alfredo.nespoli;roberto.faranda\}@polimi.it
}

\author{
Davide Poli \\ DESTEC \\ University of Pisa \\ Pisa, Italy \\ davide.poli@unipi.it
}

\author{
Fabio Bassi \\ Terna Rete Italia S.p.A. \\ National Dispatcing \\ Italy \\ fabio.bassi@terna.it
}

\begin{abstract}
The large-scale diffusion of renewable power generators contributes to trigger a crisis in power system components that started to operate closest to their thermal limits, thus increasing the risk of network congestions. To manage with these contingencies induced by Renewable Energy Sources generators, the Transmission System Operators have to implement proper corrective actions. Transmission System Operators usually adopts well-known physical methods, based on weather forecasts, to estimate the temperature of the lines in order to avoid an expensive installation of monitoring devices on their network. However, this estimation often results in inaccurate forecasts of the conductors' temperature, due to many complexities and parameters which should be considered in the physical model of the line or to not precise weather forecasts. This paper proposes an innovative method based on Artificial Neural Network to evaluate the conductor's temperature and consequently the Dynamic Thermal Rating in a given overhead line. The results based on real case studies and measures, clearly show the effectiveness and the potential of the proposed method.
\end{abstract}

Index Terms-DTR, Thermal estimation, CIGRE thermal model, ANN, Overhead line

\section{INTRODUCTION}

The large-scale diffusion of renewable power generators all over the transmission and distribution networks is leading a global transition from a centralized to a distributed generation. In particular, the inherent unpredictability of the generation of wind and photovoltaic power systems has required the system operators to schedule more reserve resources for the instantaneous balancing between the power produced by the generators and the one requested by the loads, including system losses.

The increasing penetration of renewable energy sources (RES) in power systems, together with the decentralization of the energy production, is requiring increasing capacity of the electrical grid in order to integrate such a variable power generation. Therefore, in order to avoid exceeding the degradation of the mechanical properties of the conductors and to respect phase-to-ground clearances, maximum current in the lines must be limited [1] [2] [3] [4].

It is well known that reliable OverHead Line (OHL) operation requires, amongst other things, the rigorous satisfaction of proper thermal constraints, which limit the conductor temperature in order to keep the line sag in fixed allowable ranges, avoiding the risk of ground faults. The transmission capacity of an OHL is the current (ampacity) that corresponds to the maximum acceptable temperature of conductors. Hence the maximum acceptable temperature is limited by the thermal performance of the material. Therefore it is extremely useful to give an accurate evaluation of the temperature of any line in the grid, namely Dynamic Thermal Rating (DTR) of the line. DTR, also referred to as Dynamic Line Rating (DLR), can be done by directly monitoring the temperatures of conductors, or uses weather and load forecasting to estimate their future trend, in order to calculate the actual capacity of a transmission line [5] [6]. Therefore, this can be achieved either by means of direct measurements or by an effective estimation method. The former implies a very expensive solution which is not adopted, while the latter is cheaper but often can be not very accurate. Indeed, the adopted method has to consider several parameters affecting the temperature of the line and, at the same time, should be not too complicated to be used. The temperature of the conductors depends on the current in the line and on the local weather conditions [7] [8] [9] [10].

The benefits deriving by the application of DTR techniques in existing power systems have been assessed in several papers, which proposed different solution methodologies, including those based on first-order components thermal models, or using distributed sensors for the direct measurement of the conductor temperature [11].

These techniques, if integrated in advanced optimization frameworks, known as Weather Condition-based Optimal Power Flow (W-OPF) or Electro-Thermal OPF (ET-OPF), could reliably improve the components loadability, enhancing the congestion management flexibility, and maximizing the RES generators exploitation [12] [13] [14] [15].

The goal of this work is to compare the performances of different methods for the forecast of the temperature of OHL. The comparison is made between a physical model (CIGRE) [1] [16], and a statistical method, based on Artificial Neural Networks (ANN) [17]. Such comparison is of particular interest, especially when the forecasted wind speed is lower than $2 \mathrm{~m} / \mathrm{s}$, which is proven to be the range where physical models provide a worse result [18]. 


\section{DTR ESTIMATION MODELS}

In the scientific literature it is possible to find several DTR estimation models, in the following the physical CIGRE model and a novel technique based on ANN, are presented.

\section{A. Physical CIGRE model}

In deterministic weather-based DTR procedures, the temperature of conductors is estimated using a proper thermal model of the transmission line, usually the one developed by CIGRE [7] or by IEEE [8].

Conductor's temperature depends on various factors such as conductor material, diameter, superficial characteristics, load current, and weather conditions insisting on it. Once these latter are known, the above mentioned standard allows to calculate the conductor's temperature given the line current or the capability, given the maximum feasible operating temperature. In physical models like CIGRE or IEEE, the thermal behavior of the conductor is assessed based on the following first order dynamic equation:

$$
\frac{d T_{c}}{d t}=\frac{1}{m c_{p}} \cdot\left[q_{s}+q_{j}-q_{c}-q_{r}\right]
$$

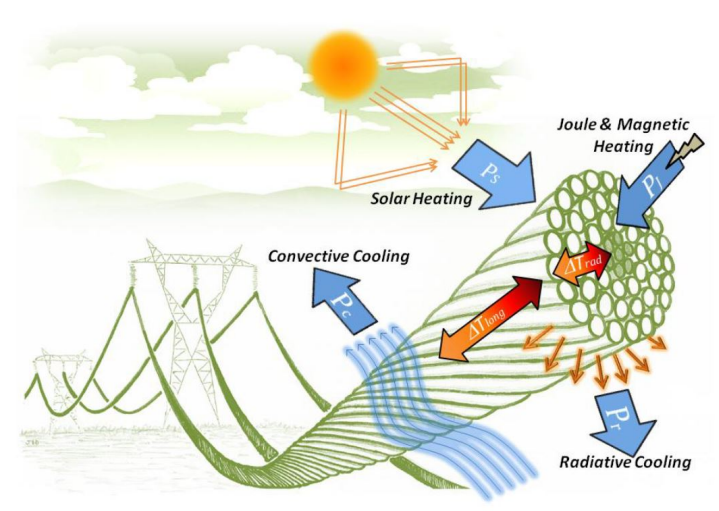

Fig. 1. Thermal behavior of OHL Conductor [7]

where:

$m c_{p}$ is the conductor heat capacity per unit length, defined as the product of its specific heat capacity, $c_{p}$, and the mass per unit of length, $m$. If the conductor consists of different materials, its heat capacity is the sum of heat capacities of all the $i_{t h}$ strands, as $m c_{p}=\sum_{i} m_{i} \cdot c_{p, i}$.

$q_{c}$ is the convected heat loss rate per unit of length. Its calculation requires a different expression depending on the wind speed.

When the wind speed is different from zero, forced convection occurs and the convective heat losses can be computed through equation 2 and 3.

$$
\begin{gathered}
q_{c 1}=K_{\text {angle }} \cdot\left(1.01+1.35 \cdot R e^{0.52}\right) \cdot k_{f} \cdot\left(T_{c}-T_{a}\right) \\
q_{c 2}=K_{a n g l e} \cdot\left(0.754 \cdot R e^{0.6}\right) \cdot k_{f} \cdot\left(T_{c}-T_{a}\right)
\end{gathered}
$$

where $R e$ is the Reynolds number, $T_{a}$ the air temperature and $T_{c}$ the conductor core temperature. While $q_{c 1}$ applies for low wind speed, $q_{c 2}$ is for high wind speed. To properly choose the correct one, for any wind speed both of them have to be computed and the largest one has to be used [8].

The term $K_{\text {angle }}$ accounts for the dependance of the convective heat loss rate on the wind direction and it is calculated as:

$$
K_{\text {angle }}=1.194-\cos (\phi)+0.194 \cdot \cos (2 \phi)+0.368 \cdot \sin (2 \phi)
$$

where $\phi$ represents the angle between the wind direction and the conductor axis.

When the wind speed is zero, natural convection occurs and the rate of heat loss can be computed as following:

$$
q_{c n}=3.645 \cdot \rho_{f}^{0.5} \cdot D_{0}^{0.75} \cdot\left(T_{c}-T_{a}\right)^{1.25}
$$

In order to compute the air density, air viscosity and air thermal conductivity are estimated at the temperature $T_{\text {film }}=$ $0.5 \cdot\left(T_{c}+T_{a}\right)$ through the polynomial expressions:

$$
\begin{gathered}
\mu_{f}=\frac{1.458 \cdot 10^{-6} \cdot\left(T_{\text {film }}+273\right)^{1.5}}{T_{f i l m}+383.4} \\
\rho_{f}=\frac{1.293-1.525 \cdot 10^{-4} \cdot H_{e}+6.379 \cdot 10^{-9} \cdot H_{e}^{2}}{1+0.00367 \cdot T_{\text {film }}}
\end{gathered}
$$

$k_{f}=2.424 \cdot 10^{-2}+7.477 \cdot 10^{-5} \cdot T_{f i l m}-4.407 \cdot 10^{-9} \cdot T_{f i l m}^{2}$

$q_{r}$ is the radiated heat loss rate per unit length, calculated as:

$$
q_{r}=17.8 \cdot D_{0} \cdot \varepsilon \cdot\left[\left(\frac{T_{c}+273}{100}\right)^{4}-\left(\frac{T_{a}+273}{100}\right)^{4}\right]
$$

where $\varepsilon$ is the conductor emissivity, which depends on the age of the conductor, and ranges between 0.27 to 0.95 , with a value of 0.5 proposed for example by IEEE, and $D_{0}$ is the external conductor diameter.

$q_{s}$ is the solar heat gain rate per unit length and is given by the expression:

$$
\begin{gathered}
q_{s}=\alpha \cdot Q_{s e} \cdot \sin (\theta) \cdot D_{0} \\
\theta=\operatorname{arcos}\left[\cos \left(H_{c}\right) \cdot \cos \left(Z_{c}-Z_{l}\right)\right]
\end{gathered}
$$

and $H_{c}$ is the solar altitude, $Z_{c}$ the solar azimuth, and $Z_{l}$ the azimuth of line. Corrected rate of solar heat gain as a function of the altitude, $Q_{\text {se }}$, equals $K_{\text {solar }} \cdot Q_{s}$, where $K_{\text {solar }}=A+$ $B \cdot H_{e}+C \cdot H_{e}^{2}$, and:

$$
\left\{\begin{array}{c}
A=1 \\
B=1.148 \cdot 10^{-4} \\
C=-1.108 \cdot 10^{-8}
\end{array}\right.
$$

$Q_{s}$ is given, for a clear or industrial atmosphere, by a polynomial expression in $H_{c}$ : 


$$
Q_{s}=A+B \cdot H_{c}+C \cdot H_{c}^{2}+D \cdot H_{c}^{3}+E \cdot H_{c}^{4}+F \cdot H_{c}^{5}+G \cdot H_{c}^{6}
$$

where the constants $A, B, C, D, E, F, G, H$ are shown in Table I

TABLE I

POLYNOMIAL COEFFICIENTS FOR $Q_{s}$

\begin{tabular}{cccc}
\hline \hline \multicolumn{2}{c}{ Clear atmosphere } & \multicolumn{2}{c}{ Industrial atmosphere } \\
\hline$A$ & -42.2391 & $A$ & 53.1821 \\
$B$ & 63.8044 & $B$ & 14.2110 \\
$C$ & 1.9220 & $C$ & $6.6138 \cdot 10^{-1}$ \\
$D$ & $3.46921 \cdot 10^{-2}$ & $D$ & $-3.1658 \cdot 10^{-2}$ \\
$E$ & $-3.61118 \cdot 10^{-4}$ & $E$ & $5.4654 \cdot 10^{-4}$ \\
$F$ & $1.94318 \cdot 10^{-6}$ & $F$ & $-4.3446 \cdot 10^{-6}$ \\
$G$ & $-4.07608 \cdot 10^{-9}$ & $G$ & $1.3236 \cdot 10^{-8}$ \\
\hline \hline
\end{tabular}

Finally, $q_{j}$ are joule heat losses, i.e. the contribution of thermal energy loss for Joule effect. This term depends on the electric resistance of the conductor at the temperature $T_{\text {avg }}$ and on the square of line current $I$ :

$$
q_{j}=I^{2} \cdot R\left(T_{c}\right)
$$

The conductor resistance $R\left(T_{c}\right)$ depends on the system frequency, the line current and the temperature. In the CIGRE and in the IEEE standards, the electric resistance is assumed to change only with respect to temperature $T_{c}$. Given a value of electric resistance for high temperatures, $T_{h}$, and one for low temperatures, $T_{l}$, it is possible to derive a linear model for electric resistance as a function of conductor temperature:

$$
R\left(T_{c}\right)=\left[\frac{R\left(T_{h}\right)-R\left(T_{l}\right)}{T_{h}-T_{l}}\right] \cdot\left(T_{c}-T_{l}\right)+R\left(T_{l}\right)
$$

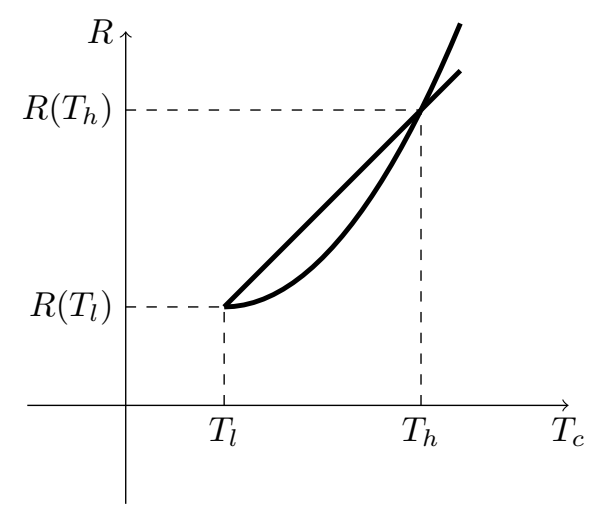

Fig. 2. Resistance-Temperature of the conductor trend

Actually, the resistance of the conductor increases with temperature somewhat faster than the linear model as shown in figure 2. This means that, if one calculates the resistance for a value of $T_{a v g}$ between $T_{l}$ and $T_{h}$, the value of resistance calculated with the linear model will be greater than the real one, and so it is conservative for the conductor temperature calculation. Instead, if we calculate the resistance for $T_{a v g}$ larger than $T_{h}$, the calculated value of resistance will be lower than the real one, whence an estimation of a non-conservative conductor temperature value. For this reason equation 15 is commonly adopted for a conductor temperature lower than $175{ }^{\circ} \mathrm{C}$. Resistance value has to include variations due to Skin Effect, Magnetic Core Effect and Radial Temperature Gradient in the conductor.

\section{B. Statistical ANN based model}

Artificial Intelligence (AI) has been long used by the scientific community in several branches. Among the different forecasting techniques, Artificial Neural Network (ANN) is one of the most used [19] [20]. The basic structural and functional unit of ANN is the "neuron". The inputs of each $k^{t h}$ neuron $x_{i}$ are multiplied by some properly tuned parameters called weights $w_{k, i}$ then summed with the neuron bias $b_{k}$, as shown in Figure 3. Finally, the output $y_{k}$ is produced through an activation function $f$ by means of the following equation:

$$
y_{k}=f\left(\sum_{i}\left(x_{i} \cdot w_{k, i}+b_{k}\right)\right)
$$

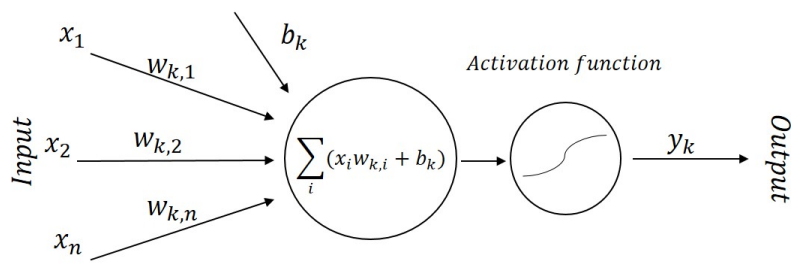

Fig. 3. Neuron model

Given its ability to generalize an arbitrary complex function, a two-layer Feed Forward Neural Network (FFNN) is adopted in this work. For this type of architecture, neurons are organized in layers and each layer receives in input the output of the previous layer and provides its output to the following one as in figure 4. To properly choose its size in terms of number of neurons, a sensitivity analysis must be performed [21]. The weights among the layers are initially randomly chosen, then they have to be optimized using a procedure called training; as explained and proven in [21], to reduce the error, it is best to adopt an ensemble forecast. Hence, the algorithm is run independently several times (trials). Each trial requires then a newly initialized FFNN, trained randomly allocating the available samples between training and validation set, keeping only their numerosity constant. Finally, the obtained results are averaged. The number of trials is then a parameter to be optimized. A trade off between the increase of the performance and the computational burden is necessary.

\section{FORECASTING METHODS APPLICATION}

The two previously described methods were implemented and tested to estimate the conductor's temperature of an Italian 


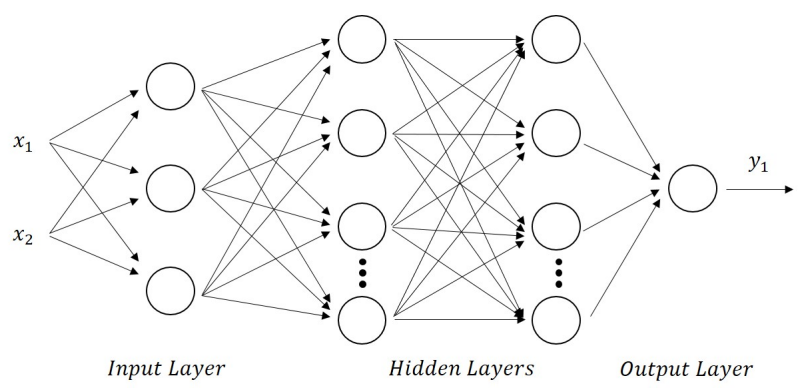

Fig. 4. Example of a generic Feed Forward Neural Network architecture

Transmission OHL. Further details on the line such as voltage and current are intentionally not provided on TSO request, without loosing the generality and the validity of the proposed comparison.

The CIGRE model is able to forecast the conductor temperature providing the weather parameters and the OHL variables together. The coefficients of the thermal model are available in literature and do not require to be furtherly tuned. The main drawback of this physicalmodel is that with wind speed lower than $2 \mathrm{~m} / \mathrm{s}$, the accuracy of the model is highly jeopardized. On the other hand, ANNs learn main trends and correlations among the different parameters, therefore they are able to adapt to varying conditions and to capture correlations not explicated in the physical model, provided that they are properly trained with historical data.

The experimental data employed in this analysis refer to the time period of one month in which the following weather forecast were available:

- Day of the Year

- Hour of the Day

- Ambient Temperature $(K)$

- Wind Speed $(\mathrm{m} / \mathrm{s})$

- Wind Direction $\left(^{\circ}\right)$

- Global Solar Radiation $\left(W / m^{2}\right)$

- Atmospheric Pressure (bar)

- Relative Humidity (\%)

In the same time frame, the temperature of the OHL was measured every 5 minutes.

For a coherent sampling of all the considered parameters, the hourly mean values were calculated. In the following section, the comparison has been performed only for the 64 samples with wind speed below $2 \mathrm{~m} / \mathrm{s}$. These parameters represent the input of both methods compared in this paper for the temperature of the conductor estimation.

As CIGRE method is a physical model, the temperature estimation for each sample can be obtained directly by following the procedure described in II-A, while for the ANN method a tuning of the statistical model parameters must be performed in advance, as previously described in II-B. In particular, a sensitivity analysis has been carried out to set the ANN parameters such as, number of neurons in the hidden layers, number of trials in the ensemble forecast and samples amount in the training and validation set respectively. In this work $90 \%$ of the available samples are assigned to the training set, while the remaining $10 \%$ were devoted to validate the model similarly to what is performed in [22]; 40 trials were adopted in the ensemble forecast of an ANN with 8 neurons in each of the two hidden layers.

\section{Preliminary Results}

In this section, 64 hourly samples with a wind speed lower than $2 \mathrm{~m} / \mathrm{s}$ are used for the comparison of the two estimation methods.

The absolute errors made by the two forecasting methods, expressed by equation 17, are compared in Figure 5:

$$
\left|\Delta T_{h}\right|=\left|T_{m, h}-T_{f, h}\right|
$$

where $T_{m, h}$ is the measured temperature of the conductor at time $h$ and $T_{f, h}$ is the forecast temperature, obtained either with the physical or the statistical method, at the same time $h$.

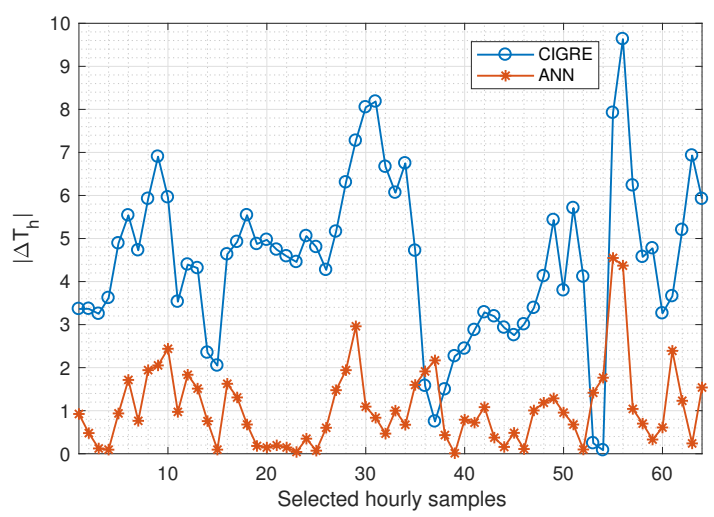

Fig. 5. Comparison of the absolute errors committed by the analysed methods

In Figure 5 it is possible to see that the ANN method provides a very good estimation performance with respect to the CIGRE method, giving a maximum absolute error equal to $4.55^{\circ} \mathrm{C}$. Generally speaking, this is largely below the one made by the physical model. In Figure 6 the outcomes of the simulations are shown. To better highlight the comparison between the two methods, results are here presented in terms of the ratio between the statistical and the physical method. On the $\mathrm{x}$-axis the number of samples are reported, while the $y$-axis shows on a logarithmic scale the corresponding ratio $\left(R_{h}\right)$ between the errors made by $\operatorname{ANN}\left(\Delta T_{A N N, h}\right)$ and $\left(\Delta T_{C I G R E, h}\right)$, as in equation 18

$$
R_{h}=\frac{\Delta T_{A N N, h}}{\Delta T_{C I G R E, h}}
$$

The blu line in Figure 6 is drawn as a reference, being the case in which the two methods make the same forecasting error.

As it is possible to notice observing the orange line in the graph, the ANN outperforms the physical model, returning a 
more accurate result in 60 cases out of 64.

Furthermore, as far as the four remaining cases are concerned,

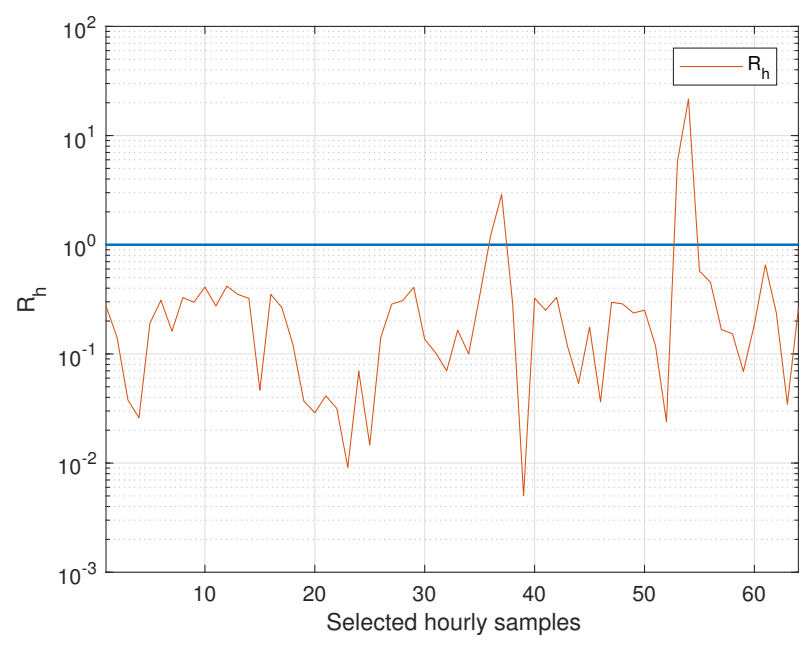

Fig. 6. Absolute errors of the Statistical method compared to the Physical as the reference

which correspond to the two positive spikes in Figure 6 the absolute error made by $\mathrm{ANN}$ is lower than $2{ }^{\circ} \mathrm{C}$ : in those specific cases, the error made by the physical method is very low, so the ratio is increased. In these cases, it must be pointed out that both estimations are very close to the measured values.

TABLE II

NUMERICAL RESULTS SHOWN IN FIGURE 6

\begin{tabular}{lccc}
\hline \hline Indicator & $\left|\Delta T_{A N N}\left({ }^{\circ} C\right)\right|$ & $\left|\Delta T_{C I G R E}\left({ }^{\circ} C\right)\right|$ & $R_{h}$ \\
\hline Min & 0.01 & 0.08 & 0.14 \\
Max & 4.55 & 9.63 & 0.47 \\
Mean & 1.06 & 4.50 & 0.24 \\
Std dev & 0.93 & 1.90 & 0.49 \\
\hline \hline
\end{tabular}

In Table II, some aggregate statistical values are provided to better compare the two methods. As it is possible to see, the temperature forecast performed with the ANN is more reliable within the described wind range, being both the average error committed and its standard deviation lower, as well as the maximum and minimum values.

\section{CONCLUSIONS}

In this work two methods for the estimation of the temperature of the conductor of a given OHL are compared. In literature the CIGRE physical model is well known and adopted, however it shows limited accuracy for peculiar wind speed conditions. For this reason an ANN has been trained and the preliminary results of the two forecasting method have been compared with wind speed less than $2 \mathrm{~m} / \mathrm{s}$.

In this condition ANN has shown a better forecasting accuracy outperforming the physical method on 60 over the 64 hourly samples that which have been considered, with an absolute mean error of $1.06{ }^{\circ} \mathrm{C}$ instead of $4.5^{\circ} \mathrm{C}$. These preliminary results encourage future works and a deeper analysis will be extended to different transmission line typologies increasing the number of cases under investigation.

\section{REFERENCES}

[1] J. R. Alvarez and C. M. Franck, "Evaluation of the accuracy of a thermal rating model to estimate the temperature of operational transmission lines," Cigrè Science\&Engineering, no. February, p. 10, 2016.

[2] L. Staszewski and W. Rebizant, "Balance Concepts for Line Ampacity Considerations," 2010.

[3] E. Mohagheghi, A. Gabash, M. Alramlawi, and P. Li, "Real-time optimal power flow with reactive power dispatch of wind stations using a reconciliation algorithm," Renewable Energy, vol. 126, pp. 509-523, 2018.

[4] E. Mohagheghi, M. Alramlawi, A. Gabash, and P. Li, "A Survey of Real-Time Optimal Power Flow," Energies, vol. 11, no. 11, p. 3142, 2018.

[5] A. Pavlinić, V. Komen, and M. Uzelac, "Application of direct collocation method in short-term line ampacity calculation," Electric Power Systems Research, vol. 155, pp. 216-224, 2018.

[6] D. Villacci, G. Giannuzzi, A. Vaccaro, C. Pisani, and E. Carlini, "Experimental deployment of a self-organizing sensors network for dynamic thermal rating assessment of overhead lines," Electric Power Systems Research, vol. 157, pp. 59-69, 2017. [Online]. Available: http://dx.doi.org/10.1016/j.epsr.2017.12.007

[7] J. Iglesias, G. Watt, D. Douglass, V. Morgan, R. Stephen, M. Bertinat, D. Muftic, R. Puffer, D. Guery, S. Ueda, K. Bakic, S. Hoffmann, T. Seppa, F. Jakl, C. Do Nascimento, F. Zanellato, and H.-M. Nguyen, Guide for thermal rating calculations of overhead lines, 2014. [Online]. Available: http://hdl.handle.net/2268/178855

[8] IEEE - PES, IEEE Standard for Calculating the Current-Temperature Relationship of Bare Overhead Conductors, 2013, vol. 2006, no. January.

[9] F. Bassi, G. M. Giannuzzi, M. Giuntoli, G. Lutzemberger, P. Pelacchi, A. Piccinin, and D. Poli, "A novel HTLS thermo-mechanical model: applications to Italian OHTL," CIGRE Session 2016, Paris, no. 21-26 August 2016, 2016.

[10] M. Giuntoli, P. Pelacchi, D. Poli, F. Bassi, and G. Giannuzzi, "Thermomechanical dynamic rating of OHTL: Applications to Italian lines," in CIGRE Session 45 - 45th International Conference on Large High Voltage Electric Systems 2014, 2014.

[11] G. Coletta, A. Vaccaro, and D. Villacci, "A review of the enabling methodologies for PMUs-based dynamic thermal rating of power transmission lines," Electric Power Systems Research, vol. 152, pp. 257-270, 2017. [Online]. Available: http://dx.doi.org/10.1016/j.epsr.2017.07.016

[12] M. Nick, O. A. Mousavi, R. Cherkaoui, and M. Paolone, "Integration of Transmission Lines Dynamic Thermal rating into real-time Optimal dispatching of power systems," Proceedings of the Universities Power Engineering Conference, vol. 2015-Novem, pp. 1-6, 2015.

[13] J. Cao, W. Du, and H. F. Wang, "Weather-Based Optimal Power Flow with Wind Farms Integration," IEEE Transactions on Power Systems, vol. 31, no. 4, pp. 3073-3081, 2016.

[14] M. X. Wang and X. S. Han, "Study on electro-thermal coupling optimal power flow model and its simplification," IEEE PES General Meeting, PES 2010, pp. 1-6, 2010.

[15] E. Mohagheghi, A. Gabash, and P. Li, "A framework for real-time optimal power flow under wind energy penetration," Energies, vol. 10, no. $4,2017$.

[16] N. P. Schmidt, "Comparison between IEEE and CIGRE ampacity standards," vol. 14, no. 4, pp. 1555-1562, 1999.

[17] M. Negnevitsky and Tan Loc Le, "Artificial neural networks application for current rating of overhead lines," Proceedings of ICNN'95 International Conference on Neural Networks, vol. 1, pp. 418-422, 1995. [Online]. Available: http://ieeexplore.ieee.org/document/488137/

[18] A. Arroyo, P. Castro, R. Martinez, M. Manana, A. Madrazo, R. Lecuna, and A. Gonzalez, "Comparison between IEEE and CIGRE thermal behaviour standards and measured temperature on a $132-\mathrm{kV}$ overhead power line," Energies, vol. 8, no. 12, pp. 13660-13671, 2015.

[19] S. H. Chen, A. J. Jakeman, and J. P. Norton, "Artificial Intelligence techniques: An introduction to their use for modelling environmental systems," Mathematics and Computers in Simulation, vol. 78, no. 2-3, pp. 379-400, 2008. 
[20] A. Nespoli, E. Ogliari, and M. Mussetta et al., "Validation of ANN Training Approaches for Day-Ahead Photovoltaic Forecasts," in 2018 International Joint Conference on Neural Networks (IJCNN), 2018.

[21] F. Grimaccia, S. Leva, M. Mussetta, and E. Ogliari, "ANN sizing procedure for the day-ahead output power forecast of a PV plant," Applied Sciences (Switzerland), vol. 7, no. 6, 2017.

[22] A. Dolara, F. Grimaccia, S. Leva, M. Mussetta, and E. Ogliari, "Comparison of Training Approaches for Photovoltaic Forecasts by Means of Machine Learning," Applied Sciences, vol. 8, no. 2, p. 228 2018. [Online]. Available: http://www.mdpi.com/2076-3417/8/2/228 